\title{
Herpes Simplex virus type 2 myeloradiculitis with a pure motor presentationin a liver transplant recipient
}

Elena Abati1*, Delia Gagliardi1*, Daniele Velardo2, Megi Meneri2, Giorgio Conte3, Claudia Cinnante3, Nereo Bresolin 1,2, Giacomo Comi 1,2, Stefania Corti1,2,4

1 Department of Pathophysiology and Transplantation (DEPT), Dino Ferrari Centre, Neuroscience Section, University of Milan, Milan, Italy.

2 Foundation IRCCS Ca' Granda Ospedale Maggiore Policlinico, Neurology Unit, Milan, Italy

3 Foundation IRCCS Ca' Granda Ospedale Maggiore Policlinico, Neuroradiology Unit, Italy

4 Corresponding author: Stefania Corti, Department of Pathophysiology and Transplantation (DEPT), Dino Ferrari Centre, Neuroscience Section, Neurology Unit, IRCCS Foundation Ca' Granda Ospedale Maggiore Policlinico, University of Milan, Milan, Italy. E-mail: stefania.corti@policlinico.mi.it

*These authors equally contributed to the work

\begin{abstract}
In this case report, we describe the first PCR-confirmed case of HSV2 myeloradiculitis with a purely motor presentation, occurring in a 68-year-old liver transplant recipient. The patient reported ascending weakness with no sensory nor sphincteric symptoms, thereby resembling acute demyelinating inflammatory neuropathy, or Guillain-Barré syndrome. HSV2 was detected in cerebrospinal fluid by PCR, and the patient was successfully treated with intravenous Acyclovir.
\end{abstract}

\section{Keywords:}

Herpes simplex virus type 2; HSV2 infection; Elsberg syndrome; myeloradiculitis; ascending weakness; liver transplant. 
Introduction

Elsberg syndrome is an extremely rare lumbosacral infectious disorder characterized by involvement of the cauda equina and associated with Herpes Simplex virus type 2 (HSV2) reactivation or, infrequently, primary infection ${ }^{1,2}$. Only 12 PCR-confirmed cases have been reported to date ${ }^{2-6}$, and they all featured sphincterial and/or sensorimotor symptoms, while a purely motor presentation is not typical and occurs rarely. HSV2 disseminated infection is known to occur in transplant recipients as a result of reactivation of dormant virus or, rarely, in a donor-derived fashion, although a neurologic involvement has never been described ${ }^{7,8}$. We hereby describe the case of a 68-year-old immunocompromised man with progressive motor impairment due to HSV2 central nervous system (CNS) infection..

\section{Clinical case}

The patient was admitted to our Emergency Department for a six-days history of progressive limb weakness. He reported a recent gastrointestinal illness with watery diarrhea, which lasted five days and disappeared after treatment with metronidazole. Six days after the resolution of the diarrhea, he started to experience progressive weakness of the lower limbs, with impairment in walking and climbing stairs, associated with lumbar pain and fatigue. He denied both sensory and autonomic or sphincteric deficits. Four days later, weakness of the hands appeared.

His medical history was relevant for a liver transplant following acute HBV hepatitis at the age of 60 . Seven years later, he was diagnosed with post-transplant large B-cell intestinal lymphoma and treated with jejunoileal resection. Since then, the patient had been receiving immunosuppressive treatment with Tacrolimus and Mofetil Mycophenolate, and antiviral treatment with anti-HbS Immunoglobulins and entecavir.

Upon admittance, physical examination was unremarkable while neurological examination showed symmetric upper and lower limb weakness with proximal predominance, steppage gait, normal sensory testing and no sphincterial involvement. The remaining neurological examination, including cranial nerves, muscle tone, deep tendon reflexes (DTRs) and plantar cutaneous response, was normal. Blood tests were normal, except for leukocytosis (12.000 wbc/mm3) and elevated C-reactive protein (6 mg/dL). An urgent lumbar puncture was performed, revealing lymphocytic pleocytosis (250 cells/mm3, with Tlymphocyte prevalence), and normal proteins and glucose levels. Virologic analysis by PCR technology disclosed the presence of HSV-2 DNA (1700 copies of viral DNA) in cerebrospinal fluid (CSF). HSV1, VZV, EBV, CMV, HHV6, adenoviruses, enteroviruses, parvoviruses, Treponema Pallidum DNA were absent in the CSF. The patient reported no history of prior genital ulcers, and at the moment of evaluation there were no signs nor symptoms of genital HSV2 infection. 
Nerve conduction studies (NCS) showed normal amplitude, conduction velocity and distal latency in motor and sensory nerves at lower limbs, with preservation of F-waves (Table 1). Electromyography revealed reduced spatial recruitment in bilateral tibialis anterior, medial gastrocnemius and vastus medialis, along with increased amplitude and length of motor unit potentials (Table 1).

Given the history of PTLD and the immunosuppression status, we decided to perform further investigations to exclude a possible reactivation. Cytologic and cytofluorimetric analysis on CSF showed mixed lymphoid cellularity, not consistent with the diagnosis of non-Hodgkin B-cell lymphoma. Given the strong association of PTLD with EBV infection, we searched for EBV-DNA presence on patient's serum and CSF, which yielded negative results. Considering the strong epidemiological association of HSV-2 infection with HIV infection, we tested our patient for HIV serology, which turned out negative ${ }^{9-11}$. We also performed a brain and spine MRI with gadolinium, which showed linear enhancement of cauda equina roots and of conus terminalis profile, in association with caudal roots enlargement (Figure 1). Brain MRI was unremarkable.

The discovery of CSF HSV-2 positivity raised suspicion for HSV-2 myeloradiculitis, or Elsberg syndrome $^{1}$. Limited evidence suggests that acyclovir may be effective in the treatment of this condition ${ }^{1,4}$. Notwithstanding that some authors advocate the use of corticosteroids as the mainstay for halting immunemediated inflammation and reducing long-term sequelae ${ }^{4}$, evidence regarding their effectiveness and safety is still lacking ${ }^{1}$. Therefore, a therapeutic course with Acyclovir $10 \mathrm{mg} / \mathrm{kg}$ three times daily was started, with progressive improvement in clinical symptoms. NCS performed one week later revealed a reduction in the amplitude of the compound muscle action potential in both posterior tibial nerves, suggesting the occurrence of axonal damage in motor nerves and roots, which was expected.

A repeat lumbar puncture showed a reduction of lymphocytic count and absence of HSV-2 DNA in the CSF, thereby treatment was discontinued after 21 days. Given the potential risk for reactivation, oral prophylactic treatment with $400 \mathrm{mg}$ of oral acyclovir twice a day was started. At discharge, the patient presented an improvement in motor function, especially in distal regions, and was able to walk independently. The patient was reviewed in our clinic after two months, and his symptoms appeared to be further ameliorated. He could walk independently, stand up from sitting position with the aid of upper limbs, and climb stairs with the aid of a handrail. His motor strength was normal in the upper limbs, while a slight residual weakness could be appreciated in the lower limbs.

Discussion 
HSV-2 is a diffuse anogenital infection which may rarely cause neurological complications, especially in immunocompromised patients. Meningitis, encephalitis, and myeloradiculitis, also called Elsberg syndrome, are all potential manifestations of the disease ${ }^{1-4,12-15}$. Compared to immunocompetent patients, solid organ transplant recipients present a higher risk for HSV2 disease, and they generally show more frequent and severe clinical manifestations ${ }^{7,16}$. In most cases, symptomatic infection results from reactivation of latent virus, although primary infection from allograft or from interpersonal transmission may occur ${ }^{7,8}$. Our patient reported no history of prior genital ulcers.

Reported manifestations of visceral or disseminated HSV2 infection in transplant recipients feature hepatitis, pneumonitis, disseminated cutaneous disease, leucopenia and fever, while a CNS involvement in these patients has never been described ${ }^{7,8,16}$. However, clinical presentation in immunocompromised patient is often atypical, therefore different aetiological agents may lack typical findings that are otherwise expected.

In previously described cases of HSV2 myeloradiculitis, sexual and sphincter dysfunction, sensory symptoms and flaccid paresis in a variable distribution are usually observed, with acute or subacute onset and course ${ }^{1,3,4}$. Pure motor presentations have been described rarely. In a recent report, Shields and colleagues described a woman with severe lower limbs weakness and urinary hesitancy due to HSV2 radiculomyelitis ${ }^{17}$. In a previous series, urinary retention was reported by $77 \%$, saddle anesthesia by $50 \%$, bowel incontinence by $10 \%$ and constipation by $43 \%$ of patients ${ }^{1}$, while sensory loss affecting the limbs was reported by $80 \%$ of patients. Prodromal symptoms, such as fever, headache, photophobia, diarrhea, myalgia, and general malaise, have been described in $17 \%$ of patients in the aforementioned series ${ }^{1}$. Our patient reported watery diarrhea prior to the onset of weakness.

Myelopathy may show a gradual ascension to cervicothoracic spinal cord levels, associated, in some cases, to a necrotizing evolution with a severe prognosis ${ }^{4,9,10}$. Immunosuppressive conditions, such as diabetes, HIV infection, and neoplasms, seem to be predisposing risk factors for this peculiar variant. As regards CSF analysis, a retrospective study found that median CSF leukocyte count was $9 / \mu \mathrm{L}$, while median CSF proteins were $143 \pm 131 \mathrm{mg} / \mathrm{dL}^{2}$.

In this case, the absence of sensory and autonomic symptoms broadened the potential differential diagnoses (Table 2). Ascending weakness associated with back pain is highly suggestive for Guillain-Barré syndrome (GBS), an acute immune-mediated polyneuropathy, which causes a monophasic illness with symmetric cranial or limb weakness, sometimes accompanied by distal paresthesias. GBS typically follows a gastrointestinal infection and it is almost invariably associated with reduced DTRs, absent F waves on nerve conduction studies (NCS) and CSF albumin-cytological dissociation. The symptoms of our patient 
raised suspicion for GBS, although some features were not typical, such as the presence of normal DTRs, the preservation of $\mathrm{F}$ waves at electroneurographic studies and the elevated pleocytosis in CSF.

Furthermore, it was of paramount importance to exclude a central nervous system (CNS) recurrence of large B-cell lymphoma. Post-transplant lymphoproliferative disorders (PTLD) are lymphoid and/or plasmacytic proliferations occurring after solid organ or allogeneic hematopoietic cell transplantation as a result of immunosuppression. They are among the most serious and potentially fatal complications of transplantation. While the majority appears to be related to the presence of Epstein-Barr virus (EBV), EBV-negative disease may happen ${ }^{18}$. CNS PTLD are uncommon, and diagnostic tests include magnetic resonance imaging (MRI) with gadolinium, cerebrospinal fluid (CSF) analysis for EBV by polymerase chain reaction (PCR) and cytology with flow cytometry ${ }^{1}$. Our patient experienced a posttransplant, EBV-negative large B-cell lymphoma one year before the onset of the symptoms described, therefore a neurologic localization of the disease should be considered in the differential diagnosis, despite follow-up after surgical resection yielded negative results.

In case of clinical uncertainty, consistent MRI findings might support the diagnosis of infectious myeloradiculitis. Despite no pathognomonic or specific neuroimaging features for HSV-2 myeloradiculitis were identified, there are some typical findings ${ }^{1}$. Multiple and often discontinuous spinal cord lesions can be observed, usually with a central or ventral distribution. In addition, nerve roots and/or lower spinal cord edema with T2-hyperintensity may occur at lumbosacral MRI, accompanied by contrast enhancement in the acute phase of infection ${ }^{1,4,5}$.

To aid clinicians in the diagnosis, clinical and laboratory-supported criteria, with 5 levels of diagnostic certainty, have been proposed by Savoldi and colleagues ${ }^{1}$. These criteria require clinical, electrophysiological or MRI evidence of radiculitis for the diagnosis to be made, associated with either laboratory confirmation of viral DNA in the CSF or presence of supportive criteria and absence of exclusionary criteria. Our patient met both the criteria for both "Laboratory-supported definite” and “Clinically definite” levels of certainty.

Although no definitive evidence from controlled trials is available, antiviral treatment with Acyclovir appeared to be beneficial in retrospective studies and anecdotal reports, and should be started as early as possible ${ }^{2,4,8}$. Recommended dosage in visceral HSV2 infection is $5-10 \mathrm{mg} / \mathrm{kg}$ intravenously every 8 hours (Grade II-1) 7 . During Acyclovir therapy, kidney function needs to be monitored daily, and antiviral dose must be adjusted if creatinine clearance falls below 50 milliliters per minute. Outcome varies according to the severity of the underlying disease, with milder cases showing almost complete remission, while severe or necrotizing forms generally hesitate in permanent disability or, rarely, death ${ }^{2,4,8}$. 
Thus, this case points out that in the presence of progressive limb weakness, even in the absence of sensory levels and bladder dysfunction or fever, an infectious disease of the spinal cord cord/roots, with particular attention to herpetic infections, should be included in the differential diagnosis. Clinicians should bear in mind the extreme heterogeneity of clinical features in transplant recipients, and infections from potentially treatable agents should be thoroughly excluded. In the case series by Savoldi and colleagues, almost half of the patients meeting criteria were not checked for HSV2, even though lumbar puncture was performed, highlighting a knowledge gap in contemporary clinical practice.

In conclusion, our case expands the spectrum of manifestations associated with HSV2 CNS infection, and highlights the paramount importance of taking into account this potential cause of ascending weakness, so to avoid overlooking the diagnosis of a potentially serious, but treatable, infectious disease.

\section{Acknowledgements}

We thank the Associazione Amici del Centro Dino Ferrari for its support.

\section{Conflict of Interest}

The authors declare that they have no conflict of interest to disclose. 


\section{Figure legend}

\section{Figure 1. Spinal Magnetic Resonance Imaging (MRI)}

Fat Sat T1 MRI images post-Gad (A-B-C coronal view and E-F-G sagittal view) showing a pathological enhancement on the nerve roots of the cauda equina (white arrows). The fat suppression of the sequence allows a better detection of the finding.

\section{Table legend}

\section{Table 1. Lower limbs motor and sensory nerve conductions studies (NCS).}

First (I) and second (II) electrophysiological exams performed 6 and 13 days after the onset of progressive limb weakness, respectively. Normal amplitude, conduction velocity and distal latency in motor and sensory nerves were observed at the first NCS. F-waves were present and with normal latency. A reduction in motor amplitude of the compound muscle action potential (CMAP) in both posterior tibial nerves was found at the second NCS, suggesting the occurrence of axonal damage involving motor nerves and roots. Left sural nerves conduction was normal.

\begin{tabular}{lcc}
\hline \multicolumn{1}{c}{ Motor conduction studies } & I & II \\
\hline Right posterior tibial nerve & & \\
Distal latency (ankle) & $4.1 \mathrm{~ms}$ & $3.6 \mathrm{~ms}$ \\
Amplitude (ankle) & $7.3 \mathrm{mV}$ & $2.7 \mathrm{mV}$ \\
Amplitude (knee) & $6.4 \mathrm{mV}$ & $1.6 \mathrm{mV}$ \\
Conduction velocity & $42.9 \mathrm{~m} / \mathrm{s}$ & $43.8 \mathrm{~m} / \mathrm{s}$ \\
F-wave latency & $47.3 \mathrm{~ms}$ & $49.6 \mathrm{~ms}$ \\
Left posterior tibial nerve & & \\
Distal latency (ankle) & $4.0 \mathrm{~ms}$ & $3.6 \mathrm{~ms}$ \\
Amplitude (ankle) & $9.4 \mathrm{mV}$ & $2.0 \mathrm{mV}$ \\
Amplitude (knee) & $6.4 \mathrm{mV}$ & $1.6 \mathrm{mV}$ \\
Conduction velocity & $41.4 \mathrm{~m} / \mathrm{s}$ & $46.6 \mathrm{~m} / \mathrm{s}$ \\
F-wave latency & - & $50.2 \mathrm{~ms}$ \\
Sensory conduction studies & I & II \\
\hline Left sural nerve & & $7 \mu \mathrm{V}$ \\
Amplitude & $6 \mu \mathrm{V}$ & $46.9 \mathrm{~m} / \mathrm{s}$ \\
Conduction velocity & $48.3 \mathrm{~m} / \mathrm{s}$ & \\
\hline
\end{tabular}


Normal values. Posterior tibial nerve: distal latency $<6 \mathrm{~ms}$; amplitude $>4 \mathrm{mV}$; conduction velocity $>42$ $\mathrm{m} / \mathrm{s}$; F-wave latency $<58 \mathrm{~ms}$. Sural nerve: amplitude $>4 \mathrm{mV}$; conduction velocity $>44 \mathrm{~m} / \mathrm{s}$.

Table 2. Differential diagnosis of acute bilateral weakness

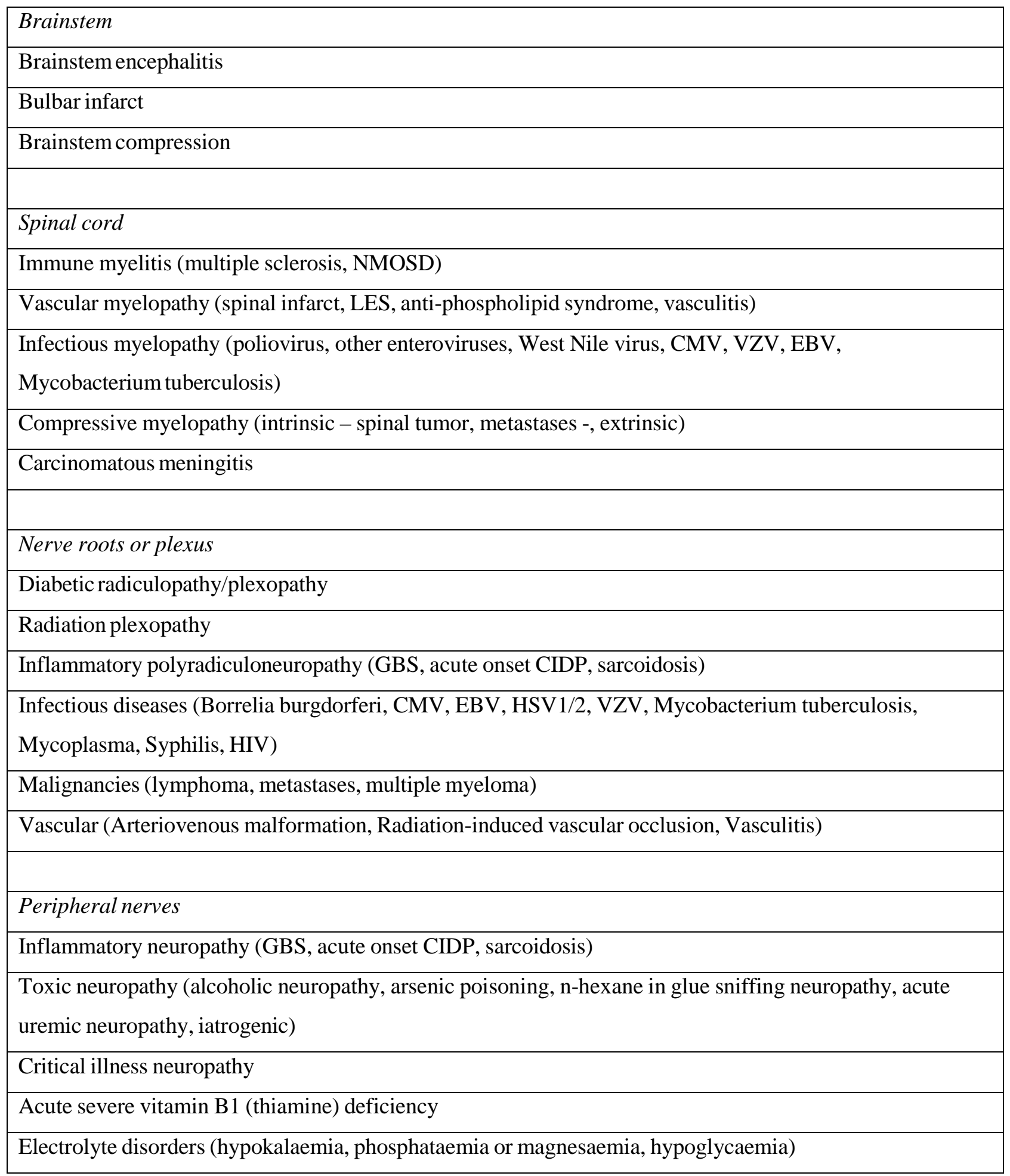




\begin{tabular}{|l|}
\hline Infectious neuropathy (Borrelia burgdorferi, tick paralysis, HIV) \\
\hline Acute intermittent porphyria \\
\hline Vasculitis \\
\hline Neuromuscular junction \\
\hline Myasthenia \\
\hline Botulism \\
\hline Acute organophosphate poisoning \\
\hline Lambert-Eaton myasthenic syndrome \\
\hline \\
\hline Muscles \\
\hline Acute inflammatory myopathy \\
\hline Critical illness myopathy \\
\hline Periodic hypo/hyperkaliemic palsy \\
\hline
\end{tabular}

Abbreviations: CIDP, chronic inflammatory demyelinating neuropathy; CMV, Cytomegalovirus; EBV, Epstein Barr virus; GBS, Guillain-Barrè syndrome; HIV, Human immunodeficiency virus; HSV, Herpes simplex virus; LES, Lupus erythematosus systemicus; NMOSD, Neuromyelitis optica spectrum disorders; VZV, Varicella zoster virus. 


\section{References}

1. Savoldi F, Kaufmann TJ, Flanagan EP, Toledano M, Weinshenker BG. Elsberg syndrome. Neurol Neuroimmunol Neuroinflammation. 2017;4(4):e355. doi:10.1212/NXI.0000000000000355

2. Eberhardt O, Küker W, Dichgans J, Weller M. HSV-2 sacral radiculitis (Elsberg syndrome). Neurology. 2004;63(4):758-759. http://www.ncbi.nlm.nih.gov/pubmed/15326269. Accessed January 27, 2019.

3. Nardone R, Versace V, Brigo F, et al. Herpes Simplex Virus Type 2 Myelitis: Case Report and Review of the Literature. Front Neurol. 2017;8:199. doi:10.3389/fneur.2017.00199

4. Nakajima H, Furutama D, Kimura F, et al. Herpes simplex Virus Myelitis: Clinical Manifestations and Diagnosis by the Polymerase Chain Reaction Method. Eur Neurol. 1998;39(3):163-167. doi:10.1159/000007927

5. Ellie E, Rozenberg F, Dousset V, Beylot-Barry M. Herpes simplex virus type 2 ascending myeloradiculitis: MRI findings and rapid diagnosis by the polymerase chain method. $J$ Neurol Neurosurg Psychiatry. 1994;57(7):869-870. http://www.ncbi.nlm.nih.gov/pubmed/8021686. Accessed January 28, 2019.

6. NAKAJIMA H, FURUTAMA D, KIMURA F, et al. Herpes Simplex Virus Type 2 Infections Presenting as Brainstem Encephalitis and Recurrent Myelitis. Intern Med. 1995;34(9):839-842. doi:10.2169/internalmedicine.34.839

7. Zuckerman R, Wald A. Herpes Simplex Virus Infections in Solid Organ Transplant Recipients. Am J Transplant. 2009;9:S104-S107. doi:10.1111/j.1600-6143.2009.02900.x

8. Singh N, Dummer JS, Kusne S, et al. Infections with Cytomegalovirus and Other Herpesviruses in 121 Liver Transplant Recipients: Transmission by Donated Organ and the Effect of OKT3 Antibodies. J Infect Dis. 1988;158(1):124-131. doi:10.1093/infdis/158.1.124

9. Bradley J, Floyd S, Piwowar-Manning E, et al. Sexually Transmitted Bedfellows: Exquisite Association Between HIV and Herpes Simplex Virus Type 2 in 21 Communities in Southern Africa in the HIV Prevention Trials Network 071 (PopART) Study. J Infect Dis. 2018;218(3):443-452. doi:10.1093/infdis/jiy178

10. Seay K, Khajoueinejad N, Zheng JH, et al. The Vaginal Acquisition and Dissemination of HIV-1 Infection in a Novel Transgenic Mouse Model Is Facilitated by Coinfection with Herpes Simplex Virus 2 and Is Inhibited by Microbicide Treatment. Doms RW, ed. J Virol. 2015;89(18):9559-9570. doi:10.1128/JVI.01326-15

11. del Mar Pujades Rodríguez M, Obasi A, Mosha F, et al. Herpes simplex virus type 2 infection increases HIV incidence: a prospective study in rural Tanzania. AIDS. 2002;16(3):451-462. http://www.ncbi.nlm.nih.gov/pubmed/11834958. Accessed January 22, 2019.

12. Tyler KL. Herpes simplex virus infections of the central nervous system: encephalitis and 
meningitis, including Mollaret’s. Herpes. 2004;11 Suppl 2:57A-64A.

http://www.ncbi.nlm.nih.gov/pubmed/15319091. Accessed January 20, 2019.

13. ELSBERG, CA. Experiences in spinal surgery. Observations upon 60 laminectomies for spinal disease. Surg Gynecol Obs. 1913;16:117-132. https://ci-nii-ac-

jp.pros.lib.unimi.it:2050/naid/10020554278/. Accessed January 27, 2019.

14. Corral I, Quereda C, Navas E, Pérez-Elias MJ, Jover F, Moreno S. Sacral myeloradiculitis complicating genital herpes in a HIV-infected patient. Int J STD AIDS. 2005;16(2):175-177. doi:10.1258/0956462053057666

15. Pagliano P, Ascione T, Carleo MA, Boccia G, De Caro F, Tortora F. HIV positive patient with HSV-2 encephalitis: case report. Infez Med. 2016;24(3):245-249.

http://www.ncbi.nlm.nih.gov/pubmed/27668908. Accessed January 20, 2019.

16. Greenberg MS, Friedman H, Cohen SG, Oh SH, Laster L, Starr S. A Comparative Study of Herpes Simplex Infections in Renal Transplant and Leukemic Patients. J Infect Dis. 1987;156(2):280-287. doi:10.1093/infdis/156.2.280

17. Shields LBE, Alsorogi MS. Herpes Simplex Virus Type 2 Radiculomyelitis Disguised as Conversion Disorder. Case Rep Neurol. 2019;40202:117-123. doi:10.1159/000499701

18. Wagner HJ, Wessel M, Jabs W, et al. Patients at risk for development of posttransplant lymphoproliferative disorder: plasma versus peripheral blood mononuclear cells as material for quantification of Epstein-Barr viral load by using real-time quantitative polymerase chain reaction. Transplantation.2001;72(6):1012-1019.http://www.ncbi.nlm.nih.gov/pubmed/11579293. Accessed January 30, 2019.

\section{Author contribution statement}

Elena Abati, Delia Gagliardi, Daniele Velardo, Megi Meneri, Nereo Bresolin, Giacomo Comi and Stefania Corti took care of patient management and made decisions about patient treatment. Elena Abati and Delia Gagliardi conceived the idea, collected clinical data and wrote the manuscript and tables. Elena Abati revised the literature. Giorgio Conte and Claudia Cinnante performed the MRI examination, acquired and analyzed the images, and prepared image panel for this manuscript. Daniele Velardo and Megi Meneri collected clinical data and revised the manuscript. Nereo Bresolin, Giacomo Comi and Stefania Corti revised the manuscript. All the authors read and approved the submitted version. 


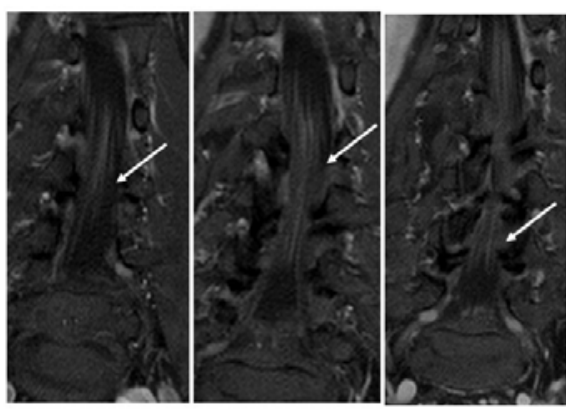

A

B

c

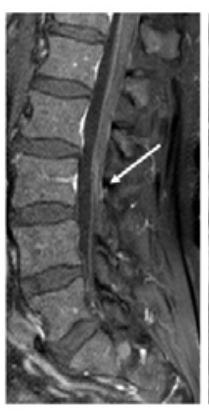

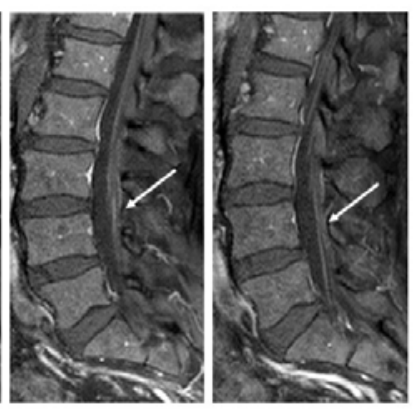

F

G

Fig. 1 